\title{
Empirical constraints for the magnitude and composition of galactic winds
}

\section{Citation}

Zahid, H. Jabran, Paul Torrey, Mark Vogelsberger, Lars Hernquist, Lisa Kewley, and Romeel Davé. 2013. "Empirical Constraints for the Magnitude and Composition of Galactic Winds." Astrophysics and Space Science 349 (2): 873-79. https://doi.org/10.1007/s10509-013-1666-0.

\section{Permanent link}

http://nrs.harvard.edu/urn-3:HUL.InstRepos:41381779

\section{Terms of Use}

This article was downloaded from Harvard University's DASH repository, and is made available under the terms and conditions applicable to Open Access Policy Articles, as set forth at http:// nrs.harvard.edu/urn-3:HUL.InstRepos:dash.current.terms-of-use\#OAP

\section{Share Your Story}

The Harvard community has made this article openly available.

Please share how this access benefits you. Submit a story.

Accessibility 


\section{Empirical Constraints for the Magnitude and Composition of Galactic Winds}

\author{
H. Jabran Zahid ${ }^{1}$ • Paul Torrey • \\ Mark Vogelsberger ${ }^{2} \cdot$ Lars Hernquist • \\ Lisa Kewley • Romeel Davé
}

\begin{abstract}
Galactic winds are a key physical mechanism for understanding galaxy formation and evolution, yet empirical and theoretical constraints for the character of winds are limited and discrepant. Recent empirical models find that local star-forming galaxies have a deficit of oxygen that scales with galaxy stellar mass. The oxygen deficit provides unique empirical constraints on the magnitude of mass loss, composition of outflowing material and metal reaccretion onto galaxies. We formulate the oxygen deficit constraints so they may be easily implemented into theoretical models of galaxy evolution. We parameterize an effective metal loading factor which combines the uncertainties of metal outflows and metal reaccretion into a single function of galaxy virial velocity. We determine the effective metal loading factor by forward-fitting the oxygen deficit. The effective metal loading factor we derive
\end{abstract}

H. Jabran Zahid

University of Hawaii at Manoa, Institute for Astronomy

${ }^{1}$ Smithsonian Astrophysical Observatory Predoctoral Fellow Paul Torrey

Harvard-Smithsonian Center for Astrophysics

Mark Vogelsberger

Harvard-Smithsonian Center for Astrophysics

${ }^{2}$ Hubble Fellow

Lars Hernquist

Harvard-Smithsonian Center for Astrophysics

Lisa Kewley

Australian National University, Research School of Astronomy and Astrophysics

Romeel Davé

Astronomy Department, University of Arizona

University of the Western Cape, Bellville

South African Astronomical Observatories

African Institute for Mathematical Sciences has important implications for the implementation of mass loss in models of galaxy evolution.

Keywords galaxies: abundances - galaxies: ISM galaxies: star-formation

\section{Introduction}

Galaxy scale winds are fundamental to galaxy evolution. The observed baryon content of galaxies is substantially below the cosmic baryon fraction (Papastergis et al. 2012). To account for this deficit, galaxy formation theories require mechanisms to reduce the efficiency with which galaxies grow (e.g., Springel and Hernquist 2003a). Consequently, strong feedback which is capable of launching galactic scale outflows is central to semi-analytic and hydrodynamical galaxy formation models (e.g., Somerville and Primack 1999; Springel and Hernquist 2003a; Schave et al. 2010; Davé et al. 2011b; Vogelsberger et al. 2013, plus many others). In simulations, energy and/or momentum injected by massive stars is capable of driving gas out of galaxies. Although these outflows are primarily required to regulate the growth of galaxies, they also drive metals out of the interstellar medium (ISM). Outflows reduce the metal content in galaxies and contribute to the enrichment of the circumgalactic and intergalactic medium (e.g. Springel and Hernquist 2003a; Oppenheimer and Davé 2006; Davé et al. 2011b).

Despite the need for outflows to regulate the growth of galaxies, the physical properties of galactic scale winds are poorly determined observationally. The lack of understanding is partly due to the complex multiphase structure of the gas, which can only be characterized in detail with observations over a broad range of wavelengths (Veilleux et al. 2005). While absorption 
line studies permit direct measurements of outflow velocities (e.g. Heckman et al. 2000; Rupke et al. 2005; Weiner et al. 2009; Chen et al. 2010; Erb et al. 2012; Martin et al. 2012; Rubin et al. 2013), estimates of the magnitude of mass loss in winds are much more difficult to obtain. Moreover, the metallicity of the wind material is constrained by only a few observations (e.g., Martin et al. 2002).

In the absence of a complete understanding of the character of outflows, theories employ wind prescriptions that are tuned to reproduce the observed properties of the galaxy population (e.g., the galaxy stellar mass function). This is typically achieved by assuming that a constant amount of energy or momentum is injected per unit star formation and that the wind speed scales in proportion to galaxy escape velocity (Martin 2005). However, in detail, the normalization and scaling of outflows vary significantly between implementations. For example, the galaxy stellar mass functions derived by Davé et al. (2011b) and Puchwein and Springel (2013) both provide satisfactory fits to the stellar mass function of local galaxies; this is in spite of mass loading factors that have normalization offsets of an order of magnitude for low stellar mass (i.e. $M_{*} \sim 10^{9} M_{\odot}$ ) systems. The uncertainties in our understanding of the physical properties of galactic winds are further complicated by numerical approximations that vary substantially from one simulation code to another (e.g Vogelsberger et al. 2012; Kereš et al. 2012; Sijacki et al. 2012; Torrey et al. 2012; Nelson et al. 2013).

Gas outflows deplete the heavy element content of galaxies. Typically it is assumed that the wind material has the same metallicity as the ambient ISM. However, the actual wind metallicity relative to the ISM may be greater if it is primarily comprised of supernova ejecta or substantially depressed if a sufficient amount of metal-poor gas is entrained as the wind propagates out of the galaxy. The total amount of metals ejected from the ISM will be proportional to the magnitude and metallicity of the outflowing gas, modulo the amount of metals ejected and subsequently reaccreted. In the absence of direct measurements characterizing these physical processes, we must rely on empirical constraints for the total metal loss in the local galaxy population to infer the properties of outflows and reaccretion.

We provide empirical constraints for outflows that can be easily implemented in galaxy formation models. In Section 2 we review the empirical oxygen mass loss estimates of Zahid et al. (2012) and the formalism for outflows typically employed in galaxy formation models. In Section 3 we introduce an effective metal loading factor which describes the loss of heavy elements from galaxies under the influence of both galactic outflows and inflows and derive the best fit for the effective metal loading factor by forward-fitting the empirical oxygen mass loss estimates. In Section 4 we directly compare our fit with parameterizations of outflows currently used in galaxy formation models and summarize our results in Section 5. We adopt a standard cosmology $\left(H_{0}, \Omega_{m}, \Omega_{\Lambda}\right)=\left(70 \mathrm{~km} \mathrm{~s}^{-1} \mathrm{Mpc}^{-1}, 0.3,0.7\right.$ and a Chabrier (2003) initial mass function (IMF).

\section{Formalism}

\subsection{The Oxygen Budget}

Estimates for the total oxygen budget of local starforming galaxies is provided by Zahid et al. (2012). We briefly summarize the formalism here. The oxygen balance can be characterized as:

$M_{T}^{o}=M_{g}^{o}+M_{*}^{o}+\Delta M^{o}$,

where $M_{T}^{o}$ is the total oxygen mass produced by stellar evolution, $M_{g}^{o}$ is the mass of oxygen currently in the ISM, $M_{*}^{o}$ is the mass of oxygen locked up in stars, and $\Delta M^{o}$ is the remaining unaccounted for oxygen which resides in the intergalactic medium (IGM) and/or circumgalactic medium (CGM). Three of the four terms in this equation can be empirically constrained.

The total mass of oxygen produced is $M_{T}^{o}=$ $y M_{*} /(1-R)$. Here, $y$ is the total mass of oxygen formed per unit mass of star formation and the factor $M_{*} /(1-R)$ is the total integrated stellar mass. This is the current mass in stars, $M_{*}$, increased by a factor $1 /(1-R)$ to account for gas recycling. Here $R$ is fraction of mass that forms into stars and is later returned back to the ISM by stellar winds and supernovae. This fraction can range between $0.2-0.5$ depending on the choice of IMF (Leitner and Kravtsov 2011; Zahid et al. 2012). In this work we adopt $R=0.35$. The mass of oxygen in the ISM is $M_{g}^{o}=Z_{g} M_{g}$, where $M_{g}$ is the galaxy gas mass and $Z_{g}$ is the gas-phase oxygen abundance. The total oxygen mass locked up in stars is

$M_{*}^{o}=(1-R) \int Z_{g}(t) \Psi(t) d t$.

Here $\Psi(t)$ is the star formation rate (SFR) as a function of time. Evaluating Equation2 2requires constraints on the chemical and star formation histories of the local star-forming galaxy population. Empirically, stellar mass growth and chemical evolution are constrained by the fact that galaxies evolve along a redshift dependent stellar mass-SFR (MS) relation, $\Psi=\Psi\left(z, M_{*}\right)$, and 
that galaxies evolve along a redshift-dependent massmetallicity (MZ) relation, $Z_{g}=Z_{g}\left(z, M_{*}\right)$.

In Zahid et al. (2012), multi-epoch observations are used to define the MS and MZ relation tracks which star-forming galaxies follow. Figures $1 \mathrm{~A}$ and $1 \mathrm{~B}$ show the stellar mass and star formation history tracks of three model galaxies assuming evolution along the MS relation. Figure $\mathbb{1 C}$ shows the gas-phase abundance tracks required by the observed $\mathrm{MZ}$ relation at several epochs and the stellar mass growth inferred from the MS relation. The mass locked up in stars is estimated by evaluating Equation 2. Using empirical estimates for $M_{T}^{o}, M_{g}^{o}$ and $M_{*}^{o}$ we can determine $\Delta M^{o}$ from Equation 1 . The oxygen deficit, $\Delta M^{o}$, represents the magnitude of oxygen mass loss that has occurred to ensure that local galaxies fall along the observed MZ relation.

Systematic uncertainties in the parameters required for conducting the oxygen census yield a range of possibilities for the oxygen deficit as a function of galaxy stellar mass (see Zahid et al. 2012). We adopt the oxygen deficit that is consistent with independent estimates of the oxygen content of hot halos of star-forming galaxies (Tumlinson et al. 2011). The empirically determined oxygen deficit is

$\Delta M^{o}\left(M_{*}\right)=4.48 \times 10^{7}\left(\frac{M_{*}}{10^{10} M_{\odot}}\right)^{1.13}\left[M_{\odot}\right]$.

A robust conclusion of Zahid et al. (2012) is that oxygen mass loss is more significant for massive galaxies. We note that the estimates of Tumlinson et al. (2011) are lower limits and the total oxygen mass in halos may be larger by a factor of a few (J. Werk, private communication; Werk et al. 2013). This would lead to a commensurate increase in the derived oxygen deficit.

The key step for parameterizing the oxygen loss for use in galaxy formation models is to cast the deficit in terms of quantities related to the galaxy potential well. We determine the halo mass of galaxies in our model, $M_{h}=M_{h}\left(z, M_{*}\right)$, using the stellar mass - halo mass relation of Behroozi et al. (2012)1. Our model of stellar mass growth taken together with the redshift dependent stellar mass - halo mass relation allows us to track the evolution of the galaxy potential well as a function of redshift. Figure 1D shows the halo mass as a function of time for the three model galaxies. The evolving virial velocity of each galaxy is easily derived from the halo mass as a function of redshift and stellar mass and is given by Equation 19 in Peeples and Shankar 2011, and references therein). In Section 3, we model the

${ }^{1}$ The relation is not directly invertible and we use tables provided by $\mathrm{P}$. Behroozi (private communication). effective metal loading factor as a function of virial velocity and forward-fit this model to the observed oxygen deficit.

\subsection{Ejecting Oxygen from Galaxies}

Our primary goal in this contribution is to provide the new empirical constraints on galaxy mass loss from Zahid et al. (2012) in a form that is directly applicable in galaxy formation models. The oxygen mass loss can be accounted for by enriched material that is ejected from the galaxy into the CGM and/or the IGM. In either case, the oxygen mass loss is caused by galactic outflows. We note that most semi-analytic models and hydrodynamic simulations of galaxy formation rely on star formation driven winds as the primary (if not only) mass loss mechanism that operates in lowmass galaxies $\left(M_{*} \lesssim 10^{11} M_{\odot}, M_{h} \lesssim 10^{12} M_{\odot}\right)$. These winds originate from deep within the galactic potential wells and are a form of "ejective" feedback. They regulate the growth of galaxies and are also thought to be primary mechanism for metal loss in star-forming galaxies. While black hole growth may also drive winds (e.g. Rupke and Veilleux 2011), we do not consider black hole driven feedback here since it is likely to be prevalent in only the most massive galaxies (e.g. Hopkins et al. 2006, 2008; Somerville et al. 2008).

A general form of the oxygen deficit integrated over the lifetime of a galaxy is

$\Delta M^{o}=\int\left[Z_{w}(t) \dot{M}_{w}(t)-Z_{a c c}(t) \dot{M}_{a c c}(t)\right] d t$,

where $Z_{w}, \dot{M}_{w}, Z_{a c c}$ and $\dot{M}_{a c c}$ are the wind metallicity, wind mass loss rate, accretion metallicity and accretion rate, respectively. None of the terms inside the integral on the right hand side of Equation 4 are well constrained observationally. In the following section we present our primary result; a parameterization of the oxygen deficit that combines the uncertainties of each of the terms in Equation 4 .

Often in galaxy formation models two basic assumptions are made regarding galactic outflows. First, it is assumed that the mass loss rate from star-formation driven winds is directly proportional to the star formation rate. This relationship is parameterized in the mass loading factor, $\eta_{w}$, such that $\dot{M}_{w}=\eta_{w} \Psi$. The mass loading factor is tuned so that models reproduce basic properties of the galaxy population. Second, the wind metallicity is parameterized by a metal loading factor, $\gamma_{w}$, such that $Z_{w}=\gamma_{w} Z_{g}$. In uniform wind models it is assumed that the metallicity of the outflowing wind material is identical to that of the ambient ISM from which the wind is launched. The metal loading factor in many models of galaxy formation is set 
to unity though there is no compelling observational evidence to suggest that this is the case.

\section{Effective Metal Loading Factor}

To take advantage of the empirical constraints on $\Delta M^{o}$ described in Section 2, we can rewrite the oxygen deficit as

$\Delta M^{o}=\int \zeta(t) \Psi(t) Z_{g}(t) d t$

where

$\zeta=\eta_{w} \gamma_{w}-\eta_{a c c} \gamma_{a c c}$

is the effective metal loading factor. In this form, the metallicity of the wind and accreted material are expressed in terms the ISM metallicity using the relative metallicity parameters $\gamma_{w}$ and $\gamma_{a c c}$. Similarly, the rate of mass ejection in the wind and the rate of mass accretion are expressed in terms of the star formation rate using the relative mass loading parameters $\eta_{w}$ and $\eta_{a c c}$. The advantage of the formulation presented in Equation 5 is that we have empirical constraints on the oxygen deficit, $\Delta M^{o}$, the metallicity, $Z_{g}$, and the star formation rate, $\Psi$, leaving only the effective metal loading factor undetermined. Thus, by adopting a functional form for the effective metal loading factor we can forward-fit the oxygen deficit to derive the best fit parameters. The key step taken here is combining our uncertainty about the mass loading, $\eta$, and relative metallicity, $\gamma$, into a single function which can be empirically constrained.

A best fit effective metal loading factor can be derived based on Equation 5 by comparing to the empirically determined oxygen deficit (see Section 2.1). To facilitate the fitting procedure, we parameterize the effective metal loading factor as

$\zeta\left(V_{v i r}\right)=\alpha\left(\frac{100 \mathrm{~km} \mathrm{~s}^{-1}}{V_{v i r}}\right)^{\beta}$

where $\alpha$ and $\beta$ set the normalization and slope, respectively. The power law functional form is adopted because a similar functional form is used to parameterize wind mass loading factors in galaxy formation models. We determine the best-fit parameter values by minimizing the residuals between the empirically derived oxygen deficit given by Equation 3 and the oxygen deficit we calculate from integrating Equation 5 using the same empirical model of stellar mass growth, halo mass growth and chemical evolution as described in Section 2.1. The best fit parameters are $\alpha=0.57$ and $\beta=0.41$ and the best-fit result for the effective
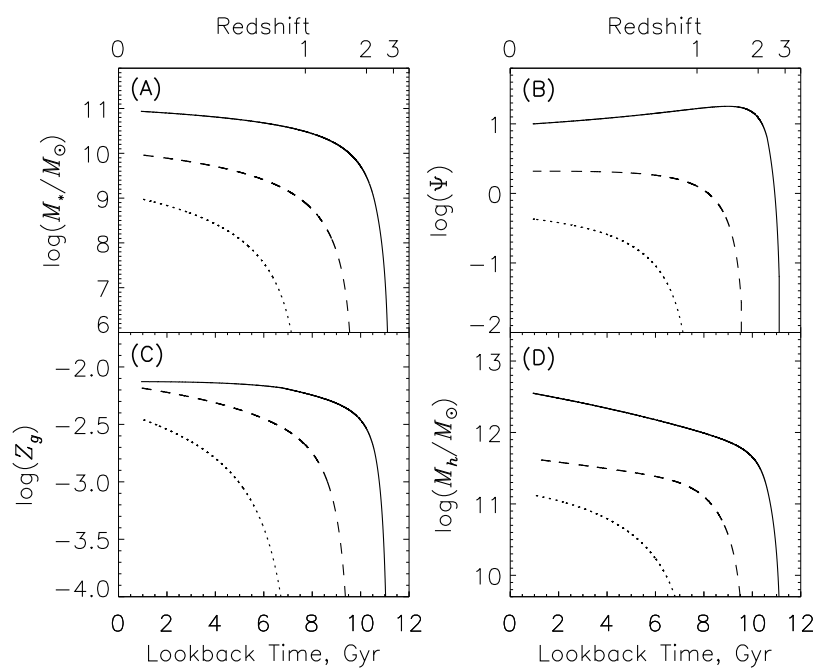

Fig. 1 Panels (A) and (B): Stellar mass and star formation history tracks for three example model star-forming galaxies as required by the multi-epoch observations of the MS relation, respectively. Panel (C): Chemical history tracks for the model galaxies determined from multi-epoch observations of the MZ relation. Panel (D): Halo mass tracks inferred from the inversion of the stellar mass-halo mass function of Behroozi et al. (2012).

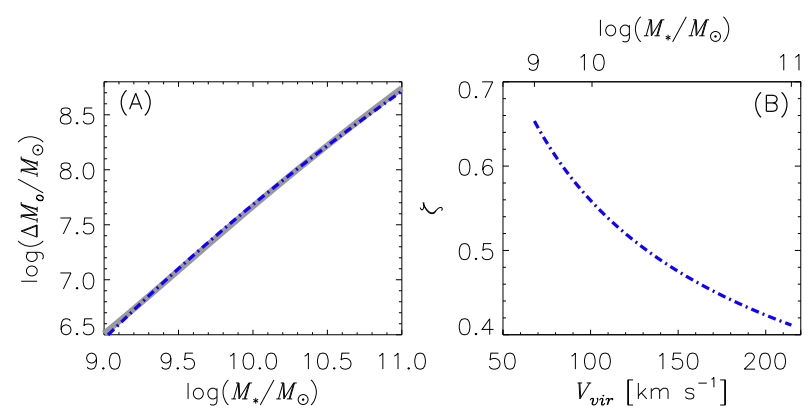

Fig. 2 Panel (A): Forward-fit of the effective metal loading factor given by Equation 7 The oxygen deficit (Equation 3) is shown by the solid grey curve. The best fit model is given by the dot-dashed blue curve. Panel (B): Effective mass loading factor derived from panel (A) plotted as a function of virial velocity. 
metal loading factor is shown by the blue dot-dashed curve in Figure 2 .

The effective metal loading factor we present can be straightforwardly applied as an empirical constraint for mass loss in simulations. Equation 6 can be solved for any factor(s) that is (are) not constrained within the simulation. If the best-fit effective metal loading factor parameterized by Equation 7 is adopted, the solution for any unconstrained factor(s) in Equation 6 will necessarily be consistent with the empirical mass loss constraints based on the oxygen deficit of Zahid et al. (2012). As empirical constraints for metal loss in galaxies improve, the approach presented here provides a straightforward methodology for implementing empirical constraints within theoretical models.

\section{Discussion}

Constraining the cycling of gas and metals in and out of galaxies is key for understanding the chemical evolution of the universe. The properties of gas cycling are defined by four quantities; the wind and accretion mass loading ( $\eta_{w}$ and $\eta_{a c c}$, respectively) and the relative metallicity of outflowing and inflowing gas with respect to the ISM metallicity $\left(\gamma_{w}\right.$ and $\gamma_{a c c}$, respectively). In the absence of direct observational constraints, the magnitude of the mass loading factors in models of galaxy formation are constrained by the baryonic mass function. The oxygen deficit, which is set by the cumulative effect of oxygen ejection and reaccretion, provides independent constraints on the metallicity of outflows and inflows. However, the magnitude and composition of both outflows and inflows are only relatively constrained by the observations and therefore are subject to significant degeneracies. In consideration of these uncertainties, the effective metal loading factor we derive combines the uncertainties of the mass loading and composition of outflows with the efficiency of metal reaccretion into a single empirically constrained function of halo virial velocity.

Table 1 Mass loading factors found in the literature and derived in this work. For simplicity, when necessary we assume $v_{w}=3 V_{v i r}$ where $v_{w}$ is the wind velocity.

\begin{tabular}{lll} 
Model & $\alpha$ & $\beta$ \\
\hline Peeples and Shankar (2011) & 0.53 & 2 \\
Vogelsberger et al. (2013) & 50 & 2 \\
Puchwein and Springel (2013) & 10 & 2 \\
Okamoto et al. (2010) & 13 & 2 \\
Davé et al. (2011b) & 2.25 & 1 \\
Hopkins et al. (2012) & 7 & 1.1 \\
\hline
\end{tabular}

Galaxies have baryonic content well below the simple prediction of the cosmological baryon fraction (e.g. Papastergis et al. 2012). Thus, massive outflows are required in simulations in order to reproduce the observed distribution of stellar masses in galaxies (Springel and Hernquist 2003b). Variable wind models, where mass loading scales with host galaxy properties, are successful at reproducing a broader range of observations (e.g. Oppenheimer and Davé 2006; Finlator and Davé 2008; Davé et al. 2011b; a); Hopkins et al. 2012; Puchwein and Springel 2013). In contrast, empirically derived mass loading factors are principally constrained by the metal content of galaxies (e.g. Peeples and Shankar 2011; Lilly et al. 2013). Figure 3A shows mass loading factors found in the literature plotted as a function of galaxy virial velocity. Mass loading factors applied in theoretical models are indicated by the black curves (i.e. Okamoto et al. 2010; Davé et al. 2011b; Hopkins et al. 2012; Puchwein and Springel 2013; Vogelsberger et al. 2013) and empirically derived mass loading factors are indicated by the colored curves (i.e. This work; Peeples and Shankar 2011; Lilly et al. 2013)2. The functions are summarized in Table 1. Based on our empirical constraints from the oxygen census, we have derived the effective metal loading factor and not the mass loading factor in Section 3. We plot the effective metal loading factor from this work in Figure $3 \mathrm{~A}$ under the assumption that $\gamma_{w}=1$ and $\eta_{w} \gamma_{w}>>\eta_{a c c} \gamma_{a c c}$ in which case $\eta_{w}=\zeta$ in Equation 6 .

Figure 3A demonstrates that there is a large discrepancy between empirically derived mass loading factors and those currently applied in theoretical models. The discrepancy results from the different observational constraints and set of assumptions adopted in theoretically and empirically motivated studies of mass loss. Theoretical mass loading factors are primarily constrained by the observed baryonic mass content of dark matter halos. Because galaxies are baryon deficient relative to the cosmological baryon fraction, massive outflows are required. However, interaction and coupling between the multi-phase outflowing material with pristine, infalling gas from the IGM is currently not well understood and this interaction may suppress initial gas accretion on galaxies (van de Voort et al. 2011). In contrast, empirically derived mass loading factors attempt to produce the observed metal content of galaxies (e.g. the MZ relation). The primary assumption being that the reaccretion of metals ejected from the galaxy ISM

\footnotetext{
${ }^{2}$ The mass loading factor derived by Lilly et al. (2013) cannot be parameterized by a power law in virial velocity (Equation 7) and therefore the model parameters do not appear in Table 1.
} 

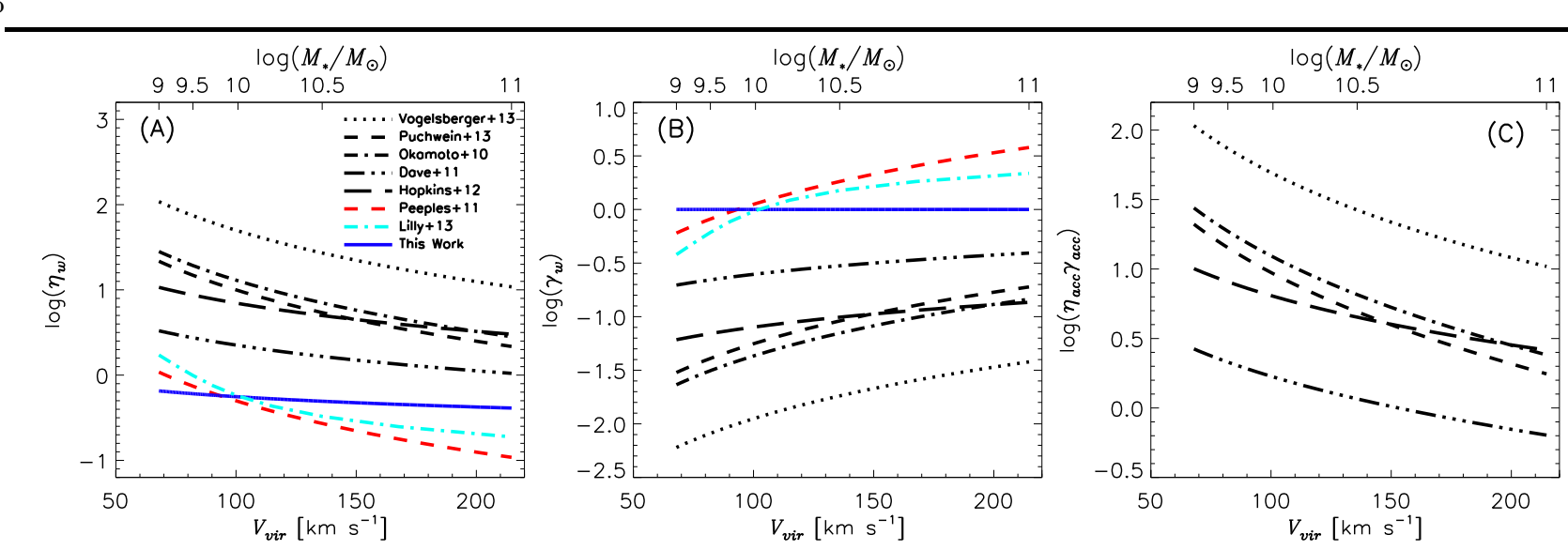

Fig. 3 (A) Comparison of various mass-loading factors compiled from the literature. The mass loading factors that are implemented in various theoretical models are shown by the black curves. The empirically derived mass-loading factors are shown by the colored curves. Under the assumption of a uniform wind and negligible metal accretion, the metal loading factor we derive reduces to the mass loading factor. The solid blue curve is our metal loading factor assuming $\gamma_{w}=1$ and $\eta_{a c c} \gamma_{a c c}<<\eta_{w} \gamma_{w}$. (B) The metallicity of outflowing material for the various mass loading factors such that galaxies are consistent with the derived oxygen deficit and galaxies in theoretical models fall on the local MZ relation under the assumption that metal reaccretion is negligible. (C) The metal reaccretion efficiency assuming a uniform wind model $\left(\gamma_{w}=1\right)$ for various mass loading factors such that galaxies are consistent with the derived oxygen deficit and fall on the local MZ relation. Under these assumptions the metal reaccretion efficiencies of (Peeples and Shankar 2011) and (Lilly et al. 2013) are negative for massive galaxies which is unphysical and so are not plotted.

is negligible. However, this is taken as a matter of convenience since efficiency of reaccretion is not observationally constrained.

In light of the observational uncertainties and varying set of assumptions, several possibilities exist for alleviating the discrepancy between theoretically and empirically derived mass loading factors :

1. Some form of feedback suppresses initial gas accretion onto dark matter halos and/or galaxies (see van de Voort et al. 2011). In this case, massive outflows are not required in order to reproduce the baryonic mass distribution of galaxies and theoretical mass loading factors in Figure 3A are unrealistically large.

2. The metallicity of outflowing gas is extremely low (i.e. $\gamma_{w}<<1$ ). While a large amounts of gas are expelled from galaxies, only a small fraction of the metals in the ISM are carried by the outflowing material. If we assume metal reaccretion is negligible (i.e. $\left.\eta_{a c c} \gamma_{a c c}<<1\right)$, then we can solve Equation 7 for the metallicity outflowing gas such that $\gamma_{w}=\zeta / \eta_{w}$. In Figure $3 \mathrm{~B}$ we plot this for the various mass loading factors. Vogelsberger et al. (2013), for example, find that they need $\gamma_{w}<1$ in order to simultaneously reproduce the galaxy stellar mass function and the MZ relation of local galaxies. The wind metallicity required in their simulations such that they are consistent with observed MZ relation of local galaxies (assuming $\eta_{a c c} \gamma_{a c c}<<1$ ) is shown by the dotted curve in Figure $3 \mathrm{~B}$.

3. Metal reaccretion is very efficient $\left(\gamma_{a c c} \eta_{a c c}>>1\right)$. Massive outflows may expel a large mass of metals but metals are able to preferentially accrete back onto galaxies (e.g. Davé et al. 2011a). Equation 7 can be solved for the metal reaccretion efficiency such that $\eta_{a c c} \gamma_{a c c}=\gamma_{w} \gamma_{w}-\zeta$. We plot the metal reaccretion efficiency in Figure $3 \mathrm{C}$ sssuming that outflowing gas has the metallicity of the ISM $\left(\gamma_{w}=1\right)$.

Most likely a combination of factors will likely contribute to resolving the discrepancy. Quantifying the properties of gas flows in Equation 4 is a key step for understanding the formation and evolution of galaxies. The effective metal loading factor derived in Section 3 provides straightforward way to apply empirical constraints in future theoretical models investigating mass loss and the apparent discrepancy.

\section{Summary and Conclusions}

We provide an important new parameterization for the empirical determination of the mass of oxygen expelled from star-forming galaxies first presented in Zahid et al. (2012). We present these constraints in the form of an effective metal loading factor which combines the uncertainties of outflows and inflows into a single 
factor. The effective metal loading factor is fit to the empirical determination of the mass of oxygen expelled from star-forming galaxies. This formulation provides joint constraints on the composition and magnitude of galaxy scale outflows. These constraints are parameterized as a function of halo virial velocity and thus may be straightforwardly implemented in theoretical models of galaxy evolution.

We show that there is large discrepancy between theoretical and empirical constraints for mass loss in galaxies. The discrepancy is due to a differing set of observational constraints and assumptions adopted in the empirical and theoretical approaches, respectively. Possibilities for resolving the discrepancy are: 1) initial gas accretion onto dark matter halos is significantly suppressed; 2) outflows are extremely metal-poor (see also Vogelsberger et al. 2013; Torrey et al. 2013); 3) metal reaccretion is extremely efficient; 4) all of the above. Our formulation of the empirical constraints on composition and magnitude of galaxy scale outflows may be implemented in theoretical models in order to explore these issues.

Our estimates of the mass of oxygen expelled from star-forming galaxies over their lives scales with galaxy stellar mass (Zahid et al. 2012). This may be a result of outflows driven by dust which operate more efficiently in massive galaxies (Zahid et al. 2013b). Recent observations of an empirical upper limit for the gas-phase abundance in galaxies that is independent of redshift suggests that wind composition does not evolve significantly (Zahid et al. 2013a). Therefore, the derived effective metal loading factor may be applicable to the galaxy population over much of cosmic time. We anticipate the empirical estimates we derive for the effective metal loading factor will be useful for constraining outflows in cosmological simulations. Future observations characterizing the physical properties of galaxy winds will provide important information for removing degeneracies between outflow composition and metal reaccretion.

Acknowledgements HJZ thanks Margaret Geller and the Smithsonian Astrophysical Observatory for the gracious hospitality enjoyed during the period when much of this work was produced. We also like to thank Peter Behroozi for help in implementing the stellar to halo mass conversion used in this work. 


\section{References}

Behroozi, P.S., Wechsler, R.H., Conroy, C.: ArXiv e-prints (2012). 1207.6105

Chabrier, G.: Publ. Astron. Soc. Pac. 115, 763 (2003). arXiv:astro-ph/0304382. doi:10.1086/376392

Chen, Y.-M., Tremonti, C.A., Heckman, T.M., Kauffmann, G., Weiner, B.J., Brinchmann, J., Wang, J.: Astron. J. 140, 445 (2010). 1003.5425. doi:10.1088/0004$6256 / 140 / 2 / 445$

Davé, R., Finlator, K., Oppenheimer, B.D.: Mon. Not. R. Astron. Soc. 416, 1354 (2011a). 1104.3156. doi:10.1111/j.13652966.2011.19132.x

Davé, R., Oppenheimer, B.D., Finlator, K.: ArXiv e-prints (2011b). 1103.3528

Erb, D.K., Quider, A.M., Henry, A.L., Martin, C.L.: Astrophys. J. 759, 26 (2012). 1209.4903. doi:10.1088/0004$637 X / 759 / 1 / 26$

Finlator, K., Davé, R.: Mon. Not. R. Astron. Soc. 385, 2181 (2008). 0704.3100. doi:10.1111/j.1365-2966.2008.12991.x

Heckman, T.M., Lehnert, M.D., Strickland, D.K., Armus, L.: Astrophys. J. Suppl. Ser. 129, 493 (2000). arXiv:astroph/0002526. doi:10.1086/313421

Hopkins, P.F., Quataert, E., Murray, N.: Mon. Not. R. Astron. Soc. 421, 3522 (2012). 1110.4638. doi:10.1111/j.1365 2966.2012.20593.x

Hopkins, P.F., Hernquist, L., Cox, T.J., Di Matteo, T., Robertson, B., Springel, V.: Astrophys. J. Suppl. Ser. 163, 1 (2006). arXiv:astro-ph/0506398. doi:10.1086/499298

Hopkins, P.F., Cox, T.J., Kereš, D., Hernquist, L.: Astrophys. J. Suppl. Ser. 175, 390 (2008). 0706.1246. doi: $10.1086 / 524363$

Kereš, D., Vogelsberger, M., Sijacki, D., Springel, V., Hernquist, L.: Mon. Not. R. Astron. Soc. 425, 2027 (2012). 1109.4638. doi:10.1111/j.1365-2966.2012.21548.x

Leitner, S.N., Kravtsov, A.V.: Astrophys. J. 734, 48 (2011). 1011.1252. doi: $10.1088 / 0004-637 X / 734 / 1 / 48$

Lilly, S.J., Carollo, C.M., Pipino, A., Renzini, A., Peng, Y.: ArXiv e-prints (2013). 1303.5059

Martin, C.L.: Astrophys. J. 621, 227 (2005). arXiv:astroph/0410247. doi:10.1086/427277

Martin, C.L., Kobulnicky, H.A., Heckman, T.M.: Astrophys. J. 574, 663 (2002). arXiv:astro-ph/0203513. doi: $10.1086 / 341092$

Martin, C.L., Shapley, A.E., Coil, A.L., Kornei, K.A., Bundy, K., Weiner, B.J., Noeske, K.G., Schiminovich, D.: Astrophys. J. 760, 127 (2012). 1206.5552. doi:10.1088/0004$637 \mathrm{X} / 760 / 2 / 127$

Nelson, D., Vogelsberger, M., Genel, S., Sijacki, D., Kereš, D., Springel, V., Hernquist, L.: Mon. Not. R. Astron. Soc. 429, 3353 (2013). 1301.6753. doi:10.1093/mnras/sts595

Okamoto, T., Frenk, C.S., Jenkins, A., Theuns, T.: Mon. Not. R. Astron. Soc. 406, 208 (2010). 0909.0265. doi:10.1111/j.1365-2966.2010.16690.x

Oppenheimer, B.D., Davé, R.: Mon. Not. R. Astron. Soc. 373, 1265 (2006). arXiv:astro-ph/0605651. doi:10.1111/j.1365 2966.2006.10989.x

Papastergis, E., Cattaneo, A., Huang, S., Giovanelli, R., Haynes, M.P.: Astrophys. J. 759, 138 (2012). 1208.5229. doi:10.1088/0004-637X/759/2/138
Peeples, M.S., Shankar, F.: Mon. Not. R. Astron. Soc. 417, 2962 (2011). 1007.3743. doi:10.1111/j.1365-2966.2011.19456.x

Puchwein, E., Springel, V.: Mon. Not. R. Astron. Soc. 428, 2966 (2013). 1205.2694. doi:10.1093/mnras/sts243

Rubin, K.H.R., Prochaska, J.X., Koo, D.C., Phillips, A.C., Martin, C.L., Winstrom, L.O.: ArXiv e-prints (2013). 1307.1476

Rupke, D.S., Veilleux, S., Sanders, D.B.: Astrophys. J. Suppl. Ser. 160, 115 (2005). arXiv:astro-ph/0506611. doi:10.1086/432889

upke, D.S.N., Veilleux, S.: Astrophys. J. Lett. 729, 27 (2011). 1102.4349. doi:10.1088/2041-8205/729/2/L27

Schaye, J., Dalla Vecchia, C., Booth, C.M., Wiersma, R.P.C., Theuns, T., Haas, M.R., Bertone, S., Duffy, A.R., McCarthy, I.G., van de Voort, F.: Mon. Not. R. Astron. Soc. 402, 1536 (2010). 0909.5196. doi:10.1111/j.13652966.2009.16029.x

Sijacki, D., Vogelsberger, M., Kereš, D., Springel, V., Hernquist, L.: Mon. Not. R. Astron. Soc. 424, 2999 (2012). 1109.3468. doi:10.1111/j.1365-2966.2012.21466.x

Somerville, R.S., Primack, J.R.: Mon. Not. R. Astron. Soc. 310, 1087 (1999). arXiv:astro-ph/9802268. doi:10.1046/j.13658711.1999.03032.x

Somerville, R.S., Hopkins, P.F., Cox, T.J., Robertson, B.E., Hernquist, L.: Mon. Not. R. Astron. Soc. 391, 481 (2008). 0808.1227. doi:10.1111/j.1365-2966.2008.13805.x

Springel, V., Hernquist, L.: Mon. Not. R. Astron. Soc. 339, 289 (2003a). arXiv:astro-ph/0206393. doi:10.1046/j.13658711.2003.06206.x

Springel, V., Hernquist, L.: Mon. Not. R. Astron. Soc. 339, 312 (2003b). arXiv:astro-ph/0206395. doi:10.1046/j.13658711.2003.06207.x

Torrey, P., Vogelsberger, M., Sijacki, D., Springel, V., Hernquist, L.: Mon. Not. R. Astron. Soc. 427, 2224 (2012). 1110.5635. doi:10.1111/j.1365-2966.2012.22082.x

Torrey, P., Vogelsberger, M., Genel, S., Sijacki, D., Springel, V., Hernquist, L.: ArXiv e-prints (2013). 1305.4931

Tumlinson, J., Thom, C., Werk, J.K., Prochaska, J.X., Tripp, T.M., Weinberg, D.H., Peeples, M.S., O'Meara, J.M., Oppenheimer, B.D., Meiring, J.D., Katz, N.S., Davé, R., Ford, A.B., Sembach, K.R.: Science 334, 948 (2011). 1111.3980. doi:10.1126/science. 1209840

van de Voort, F., Schaye, J., Booth, C.M., Haas, M.R., Dalla Vecchia, C.: Mon. Not. R. Astron. Soc. 414, 2458 (2011). 1011.2491. doi:10.1111/j.1365-2966.2011.18565.x

Veilleux, S., Cecil, G., Bland-Hawthorn, J.: Annu. Rev. Astron. Astrophys. 43, 769 (2005). arXiv:astro-ph/0504435. doi:10.1146/annurev.astro.43.072103.150610

Vogelsberger, M., Sijacki, D., Kereš, D., Springel, V., Hernquist, L.: Mon. Not. R. Astron. Soc. 425, 3024 (2012). 1109.1281. doi:10.1111/j.1365-2966.2012.21590.x

Vogelsberger, M., Genel, S., Sijacki, D., Torrey, P., Springel, V., Hernquist, L.: ArXiv e-prints, 1305.2913 (2013). 1305.2913

Weiner, B.J., Coil, A.L., Prochaska, J.X., Newman, J.A., Cooper, M.C., Bundy, K., Conselice, C.J., Dutton, A.A., Faber, S.M., Koo, D.C., Lotz, J.M., Rieke, G.H., Rubin, K.H.R.: Astrophys. J. 692, 187 (2009). 0804.4686. doi:10.1088/0004-637X/692/1/187 
Werk, J.K., Prochaska, J.X., Thom, C., Tumlinson, J., Tripp, T.M., O’Meara, J.M., Peeples, M.S.: Astrophys. J. Suppl. Ser. 204, 17 (2013). 1212.0558. doi:10.1088/00670049/204/2/17

Zahid, H.J., Dima, G.I., Kewley, L.J., Erb, D.K., Davé, R.: Astrophys. J. 757, 54 (2012). 1207.5509. doi:10.1088/0004$637 \mathrm{X} / 757 / 1 / 54$

Zahid, H.J., Geller, M.J., Kewley, L.J., Hwang, H.S., Fabricant, D.G., Kurtz, M.J.: Astrophys. J. Lett. 771, 19 (2013a). 1303.5987. doi:10.1088/2041-8205/771/2/L19

Zahid, H.J., Torrey, P., Kudritzki, R., Kewley, L., Dave, R., Geller, M.: ArXiv e-prints (2013b). 1306.1367 